\title{
Erratum to: Improved xylose fermentation of Kluyveromyces marxianus at elevated temperature through construction of a xylose isomerase pathway
}

\author{
Rongliang Wang $\cdot$ Lulu Li $\cdot$ Biao Zhang • \\ Xiaolian Gao $\cdot$ Dongmei Wang $\cdot$ Jiong Hong
}

Published online: 4 June 2013

(c) Society for Industrial Microbiology and Biotechnology 2013

\section{Erratum to: J Ind Microbiol Biotechnol \\ DOI 10.1007/s10295-013-1282-6}

In the original publication of the article, some errors were identified in Table 6 . The corrected text and table are given below

1. In Table 6: For the strain YRL005 at $42{ }^{\circ} \mathrm{C}$ with $50 \mathrm{~g} / \mathrm{l}$ xylose, in the column of Xylose consumption $(\mathrm{g} / \mathrm{l})$, the number should be $30.15 \pm 0.38$. And for the same strain at $45{ }^{\circ} \mathrm{C}$, the number in the column of Xylose consumption $(\mathrm{g} / \mathrm{l})$ should be $16.6 \pm 0.11$, and in the column of Ethanol $(\mathrm{g} / \mathrm{l})$, the number should be $5.21 \pm 0.07$. For the $S$. cerevisiae $\mathrm{H} 131-\mathrm{A} 3-\mathrm{AL}^{\mathrm{CS}}$, the Xylose consumption (g/l) should be 40 , and the temperature $\left({ }^{\circ} \mathrm{C}\right)$ should be 30 . The corrected table is given here.

2. In the Introduction: In the second page, right column, line 12 "Because $K$. marxianus 1777 could ferment with glucose rather well $(0.49 \mathrm{~g} / \mathrm{g})$ at $45^{\circ} \mathrm{C}$, the experiments at $45^{\circ} \mathrm{C}$ were used to determine if the strain RYL005 can also ferment xylose well." should be "Because K. marxianus NBRC 1777 could ferment with glucose rather well $(0.49 \mathrm{~g} / \mathrm{g})$ at $45^{\circ} \mathrm{C}$, the experiments at $45^{\circ} \mathrm{C}$ were used to determine if the strain YRL005 can also ferment xylose well."

The online version of the original article can be found under doi:10.1007/s10295-013-1282-6.

R. Wang $\cdot$ L. Li $\cdot$ B. Zhang $\cdot$ X. Gao $\cdot$ D. Wang $\cdot$ J. Hong School of Life Sciences, University of Science and Technology of China, Hefei, People's Republic of China

R. Wang $\cdot$ L. Li $\cdot$ B. Zhang $\cdot$ X. Gao .

D. Wang $(\bowtie) \cdot$ J. Hong $(\bowtie)$

Hefei National Laboratory for Physical Science at the Microscale, Hefei 230026, Anhui, People's Republic of China

e-mail: dmwang09@ustc.edu.cn

J. Hong

e-mail: hjiong@ustc.edu.cn 
Table 6 Comparison of xylose consumption and ethanol formation among recombinant yeast strains with the XI pathway

\begin{tabular}{|c|c|c|c|c|c|c|c|c|}
\hline $\begin{array}{l}\text { Xylose } \\
(\mathrm{g} / \mathrm{l})\end{array}$ & $\begin{array}{l}\text { Xylose } \\
\text { consumption } \\
(\mathrm{g} / \mathrm{l})\end{array}$ & $\begin{array}{l}\text { Temperature } \\
\left({ }^{\circ} \mathrm{C}\right)\end{array}$ & $\begin{array}{l}\text { Ethanol } \\
\text { yield }(\mathrm{g} / \mathrm{g})\end{array}$ & $\begin{array}{l}\text { Ethanol } \\
(\mathrm{g} / \mathrm{l})\end{array}$ & $\begin{array}{l}\text { Ethanol } \\
\text { productivity } \\
(\mathrm{g} / \mathrm{l} / \mathrm{h})\end{array}$ & $\begin{array}{l}\text { Time } \\
\text { (h) }\end{array}$ & Yeast & Reference \\
\hline 20 & 16 & 30 & 0.31 & 4.9 & 0.02 & 240 & S. cerevisiae LBsXI & {$[11]$} \\
\hline 50 & $27.9 \pm 0.7$ & 30 & $0.43 \pm 0.07$ & 11.9 & 0.119 & 100 & S. cerevisiae TMB 23362 & {$[31]$} \\
\hline 30 & 28.91 & 30 & 0.347 & 9.36 & 0.078 & 72 & $\begin{array}{l}\text { S. cerevisiae MT8-1 } \Delta \mathrm{GRE} 3 / \\
\text { XK } \delta \mathrm{XI}\end{array}$ & {$[43]$} \\
\hline 25 & 18 & 30 & 0.42 & 7.6 & 0.045 & 170 & S. cerevisiae BWY10Xyl & {$[5]$} \\
\hline 20 & 14.84 & 30 & 0.43 & 6.32 & 0.07 & 90 & S. cerevisiae ADAP8 & {$[25]$} \\
\hline 50 & 15.05 & 30 & 0.39 & 6.05 & 0.043 & 140 & S. cerevisiae ADAP8 & {$[25]$} \\
\hline 20 & 20 & 30 & 0.43 & 8.68 & 0.18 & 48 & S. cerevisiae RWB 217 & {$[21]$} \\
\hline 30 & 10.4 & 38 & 0.12 & 1.3 & 0.012 & 110 & S. cerevisiae $\mathrm{H} 158(\mathrm{pBXI})$ & [47] \\
\hline 40 & 40 & 30 & 0.41 & 16.4 & 0.68 & 24 & S. cerevisiae $\mathrm{H} 131-\mathrm{A} 3-\mathrm{AL}^{\mathrm{CS}}$ & {$[51]$} \\
\hline 80 & NA & 37 & NA & 1.03 & 0.011 & 96 & $\begin{array}{l}\text { H. polymorpha }{ }^{\Delta} \text { xyl1 }{ }^{\Delta} \text { xyl2- } \\
\text { A(EcxylA) \#4L/3 }\end{array}$ & [9] \\
\hline 80 & NA & 48 & NA & 0.6 & 0.006 & 96 & $\begin{array}{l}\text { H. polymorpha }{ }^{\Delta} \mathrm{xyl1}{ }^{\Delta} \mathrm{xyl} 2- \\
\text { A(EcxylA) \#4L/3 }\end{array}$ & [9] \\
\hline 50 & $30.15 \pm 0.38$ & 42 & $0.38 \pm 0.003$ & $11.52 \pm 0.019$ & 0.069 & 168 & K. marxianus YRL 005 & $\begin{array}{l}\text { This } \\
\text { study }\end{array}$ \\
\hline 50 & $16.6 \pm 0.11$ & 45 & $0.31 \pm 0.014$ & $5.21 \pm 0.07$ & 0.054 & 96 & K. marxianus YRL 005 & $\begin{array}{l}\text { This } \\
\text { study }\end{array}$ \\
\hline
\end{tabular}

Values are presented as mean and standard deviation of three independent experiments

If a reference contained several strains, only the best one is shown here. The data without the standard deviation were due to no standard deviation provided in the original paper 